\title{
Variabilité des caractères morphologiques des plantules de cinq ethno-variétés de karité (Vitellaria paradoxa C.F. Gaertn, Sapotaceae) au Burkina Faso
}

\author{
Abdoulazize SANDWIDI ${ }^{*}$, Marthe Sandrine BASSOLE-DOIGNET ${ }^{1}$, \\ Boukary Ousmane DIALLO ${ }^{1}$ et Mahamadou SAWADOGO ${ }^{2}$ \\ ${ }^{1}$ Département Environnement et Forêts, Institut de l'Environnement et de Recherches Agricoles, BP 7047 \\ Ouagadougou, Burkina Faso. \\ ${ }^{2}$ Unité de Formation et de Recherche en Sciences de la Vie et de la Terre, Laboratoire Biosciences, Université \\ Ouaga I Pr. Joseph Ki-Zerbo, BP 7021 Ouagadougou, Burkina Faso. \\ *Auteur correspondant ; E-mail: aziz.sandwidi@yahoo.fr; Tél: 0022671316079
}

RESUME

Espèce d'intérêt économique, le karité a un âge à la première mise à fruit compris entre 12 et 20 ans. Ceci rallonge son schéma de sélection. Pour lever cette contrainte il faut effectuer des tests précoces dès l'âge de la pépinière. La présente étude porte sur cinq ethno-variétés de karité identifiées au Burkina Faso. Les ethno-variétés sont des noms attribués par les paysans à différentes variantes de fruits du karité. L'objectif de l'étude est d'identifier des caractères juvéniles pertinents comme indice de sélection précoce. L'étude porte sur 100 plantules de 5 ethno-variétés. Au total 16 variables biométriques ont été mesurées au niveau des parties aériennes et souterraines. Les analyses statistiques révèlent des différences significatives entre les ethnovariétés au niveau du nombre de rameau $(P=0,04)$, du poids sec des feuilles $(P=0,02)$, de la largeur de la feuille $(P=0,03)$, la longueur du pivot $(P=0,0001)$, du poids frais des racines $(P=0,005)$ et du poids sec des racines $(P=0,014)$. De plus un compromis d'investissement des ressources est observé entre les parties aériennes et souterraines ainsi qu'entre les racines principales et secondaires. Il ressort de cette étude que les caractères discriminants au jeune âge constituent des indicateurs pour une sélection précoce des ethno-variétés. (C) 2019 International Formulae Group. All rights reserved

Mots clés: Vitellaria paradoxa, ethno-variété, Sélection précoce.

\section{Variability of morphological characters of seedlings of five ethnovarieties of shea (Vitellaria paradoxa C. F. Gaertn, Sapotaceae) in Burkina Faso}

\begin{abstract}
A species of economic interest, shea has an age at first fruit set between 12 and 20 years. This extends his selection scheme. To overcome this constraint, early tests must be performed at nursery age. This study focuses on five ethno-varieties of shea identified in Burkina Faso. Ethno-varieties are names attributed by farmers to different varieties of shea fruits. The objective of the study is to identify relevant juvenile traits as an early selection index. The study covers 100 seedlings of 5 ethno-varieties. A total of 16 biometric variables were measured in the aerial and underground parts. Statistical analyzes reveal significant differences between ethno-varieties in shoot number $(P=0.04)$, leaf dry weight $(P=0.02)$, leaf width $(P=0,03)$, pivot length $(P$ $=0.0001)$, fresh root weight $(P=0.005)$ and root dry weight $(P=0.014)$. In addition, a compromise of
\end{abstract}


investment of resources is observed between the aerial and underground parts as well as between the main and secondary roots. This study shows that early discriminant traits are indicators for early selection of ethnovarieties.

(C) 2019 International Formulae Group. All rights reserved

Keywords: Vitellaria paradoxa, ethnovariety, early selection.

\section{INTRODUCTION}

En Afrique Subsaharienne, les diverses pressions exercées sur les écosystèmes forestiers entraînent des dégâts considérables pouvant conduire à la perte progressive de certaines espèces végétales et/ou animales (Traoré, 2008 ; Thiombiano et al., 2010). Face à cette situation, il est nécessaire d'élaborer des stratégies de conservation et de domestication des espèces agro-forestières d'intérêts socio-économiques majeurs (Belem et al. 2008 ; Devineau et al., 2009). Parmi ces stratégies, la domestication des fruitiers locaux s'avère être une solution convaincante (Diallo et al., 2007; Ræbild et al., 2011). A cet effet, le Burkina Faso a adopté depuis plus de deux décennies, un plan de domestication et d'amélioration génétique des espèces locales prioritaires. L'une de ces espèces est le karité (Vitellaria paradoxa C.F. Gaertn Hepper) dont les principaux produits (amandes et beurre) ont été classés quatrième à l'exportation après l'or, le coton et le bétail (Badini et al., 2011). Cependant, l'espèce n'a toujours pas fait l'objet de plantations de production (Lamien et al., 2008 ; Asante et al., 2012) à grande échelle pour répondre de façon pérenne à la demande. Ceci s'expliquerait par son âge à la première fructification très long (12 et 20 ans) qui décourage les potentiels arboriculteurs. Diallo et al. (2010) et Weber et al. (2018) ont noté que, les caractéristiques de référence dès l'âge de la pépinière peuvent constituer des indicateurs dans le schéma de sélection pour les espèces à très longue durée de vie tel que le karité. Il est à signaler que pour les espèces locales du Sahel africain, la vigueur de croissance des plantules (hauteur et diamètre à la base) ainsi que leurs stratégies d'allocation des ressources au niveau des parties aériennes et souterraines peuvent constituer des indices forts dans la sélection (Diallo et al., 2010; Sarir et Benmahioul,
2017). Toutefois, il faut souligner que les différents programmes de domestication des ligneux sont confrontés au manque de données scientifiques sur la biologie et l'écologie des espèces cibles (Diallo, 2001). Parmi ces informations figurent les connaissances sur la variabilité phénotypique et génétique du matériel végétal. Pourtant, dans les environnements climatiques changeants, la compréhension de la variabilité phénotypique des arbres forestiers et particulièrement celle de la variabilité de la biomasse est essentielle pour établir la relation entre le phénotype végétatif et les caractères recherchés à l'âge adulte (Kobe et al., 2010 ; Poorter et al., 2012; Weber et al., 2018). C'est dans cette optique que, dans les pays en développement, des tests à long terme sous forme d'essais de provenances ont été utilisés pour étudier la variabilité de comportement des plants issus de diverses sources de semences récoltées dans différentes zones géographiques (Diallo et al., 2007 ; Bayala et al., 2009). Cette approche consiste à mettre les plants sur une parcelle d'expérimentation pour permettre l'expression des caractères spécifiques à chaque provenance traduisant ainsi une différenciation au niveau génétique. Mais, il ressort que de tels dispositifs sont coûteux aussi bien dans leur mise en place que dans leur maintien à long terme. De plus, compte tenu de la longue durée de vie des arbres forestiers comme le karité, l'expression des caractères recherchés ne se fait qu'à l'âge adulte (15 à 30 ans) rendant ainsi l'âge de la sélection aléatoire. Pour raccourcir le schéma de sélection il est important d'obtenir des informations juvéniles qui peuvent être liées aux caractères recherchés à l'âge adulte. Ceci passe nécessairement par des tests précoces, c'est-à-dire au stade où les effets maternels sont encore forts (Diallo et al., 2010). D'autres alternatives ont été utilisées par de 
nombreux auteurs dans des études comparatives du matériel végétal originaire de différentes zones écologiques en Afrique (Bayala et al., 2009; Diallo et al., 2010 ; Korbo et al., 2012 ; Bezzalla et al., 2017 ; Sandwidi et al., 2018a). Cependant, il s'agit principalement des études d'adaptation qui ne mettent pas en relief l'aspect variétal des espèces. Au Burkina Faso, des études récentes sur l'aspect variétal du karité sont parvenues à identifier et caractériser plusieurs morphotypes du karité de la sous espèce paradoxa selon les perceptions paysannes (Karambiri et al., 2017; Sandwidi et al., 2018b). Des travaux similaires réalisés en Ouganda par Gwali et al. (2011) ont permis également d'identifier et de caractériser des morphotypes de la sous espèce nilotica. Ces variantes morphologiques décrites selon la perception paysanne sont appelées des ethnovariétés. Elles sont épargnées de manière empirique et aléatoire par les paysans au moment des défriches pour l'installation des champs de cultures. De ce constat, pour disposer du matériel végétal à haute valeur génétique il serait nécessaire de pouvoir anticiper sur la sélection de ces ethno-variétés au jeune âge. Afin de mieux recommander des sources de semences qui sont appropriées aux différentes zones agro-écologiques et aux types de production et/ou de reboisement. Ainsi, la présente étude se fixe pour objectif principal d'identifier des caractères pertinents pour la sélection précoce des ethno-variétés. De façon spécifique elle vise à: (i) déterminer les caractères morphologiques discriminants propres à chaque ethno-variété, (ii) rechercher l'existence de compromis d'investissement des ressources entre les parties aériennes et souterraines des plantules ; (iii) déterminer les relations existantes entre les différents paramètres phénotypiques des ethno-variétés au stade juvénile.

\section{MATERIEL ET METHODES}

\section{Site d'expérimentation}

Les travaux ont été réalisés en saison hivernale dans la pépinière expérimentale du Département Environnement et Forêts de l'Institut de l'Environnement et de Recherches
Agricoles (INERA/DEF) du Burkina Faso. Cette pépinière est située dans la forêt classée $\mathrm{du}$ barrage $\mathrm{N}^{\circ} 3$ de Ouagadougou (1 ${ }^{\circ} 30^{\prime}$ longitude Ouest, $12^{\circ} 22^{\prime}$ latitude Nord et à 306 m d'altitude).

\section{Matériel végétal}

L'étude a porté sur des plantules de cinq ethno-variétés les plus fréquemment rencontrées dans les parcs agro-forestiers au Burkina Faso. Ces plantules sont issues des semis directs des noix récoltées dans trois parcs agro-forestiers de la grappe des villages de Yarci, Thibou et de Sibalo de la commune de Arbolé en zone nord-soudanienne. Ces trois parcs se trouvent sur des moyens glacis avec des caractéristiques pédoclimatiques quasi identiques. Il s'agit d'une mégapopulation fragmentée sous l'action du défrichement au sein de laquelle il n'y a pas interruption de flux de gènes. Ces plantules ont été produites dans des conteneurs en polyéthylène puis entretenues en pépinière après germination jusqu'à l'âge de 2 ans (Age de la plantation). Le substrat ayant servis de support de production des plantules est composé d'un mélange de terre (2 Brouettes de terre +1 brouette de sable +1 brouette de sciure de bois). Au totale 100 plantules, soit 20 plantules/ethno-variété ont été utilisées dans l'expérimentation selon un dispositif aléatoire. Ces ethno-variétés sont: Taam tolma (TT); Taam bonlogo (Tb); Taam Miidou (TM); Taam guilissi (TG) et Taam bèda (TB). Leurs caractéristiques sont répertoriées dans le Tableau 1.

\section{Méthodes}

\section{Collecte des données}

Les mesures biométriques (Figure 1) ont été effectuées sur chaque plantule à l'aide d'un pied à coulisse électronique et d'une règle graduée. Les variables mesurées sont: la hauteur de la tige (HT), le diamètre au collet (DC), la longueur et la largeur de la feuille (LF, IF) et la longueur du pétiole (LP). De plus d'autres variables ont été obtenues par comptage numérique: le nombre de ramifications (NR) et le nombre de racines secondaires (NRS). Par la suite, les feuilles, 
les tiges et les racines ont été prélevées et conservées dans des sachets plastiques étiquetés pour éviter tout dessèchement. Ces échantillons prélevés ont été acheminés au laboratoire pour effectuer d'autres mesures biométriques et des pesés à l'aide d'une balance électrique (Figure 1) afin de déterminer : la longueur et le diamètre de la racine principale (LRP, DRP), le poids frais de la tige (PFT), le poids frais de la feuille (PFF) et le poids frais des racines (PFR). Enfin, les échantillons ont été mis dans une étuve réglée à une température de $60{ }^{\circ} \mathrm{C}$ pendant $72 \mathrm{~h}$. C'est à l'issu du séchage que les échantillons ont été pesés à nouveau pour déterminer le poids sec des différentes parties: le poids sec de la tige (PST), le poids sec des feuilles (PSF) et le poids sec des racines (PSR).

\section{Analyses statistiques}

Le traitement statistique des données porte sur:

(i) une analyse de variance selon le modèle linéaire généralisé (GLM) dans lequel l'effet ethno-variété a été testé sur chaque variable qui permet d'obtenir des $\mathrm{F}$ statistiques corrigés en suivant le modèle mathématique : $Y_{\mathrm{eij}}=M+P_{\mathrm{i}}+E_{\mathrm{i}}$

Avec $Y e i$ la valeur observée de la plantule de l'ethno-variété $i ; M$ la moyenne générale de l'essai ; $P i$ l'effet de l'ethno-variété $i, i=\{1$, $2, \ldots . .5\} ; E i$ le résidu non expliqué par le modèle. Ensuite le test de Newman et Keuls au seuil de $5 \%$ a servi pour la comparaison des moyennes des différentes variables.

(ii) une analyse en composantes principales (ACP) qui a permis d'analyser simultanément toutes les variables à partir des axes principaux. Cette analyse a rendu possible la caractérisation des ethno-variétés par rapport à l'ensemble des variables.

(iii) le calcul des rapports Hauteur/diamètre et Biomasse aérienne/Biomasse souterraine (fraiche et sèche) de chaque ethno-variété a été effectué. Selon Lamhamedi et al. (1997), la qualité des plants forestiers est estimée par le ratio de robustesse. Ce ratio est obtenu par le rapport Hauteur/Diamètre (H/D) exprimé en $\mathrm{cm} / \mathrm{mm}$ qui doit être inférieur à 7 .

(iv) Deux régressions linéaires ont été effectuées : il s'agit de la relation entre le poids frais des feuilles et celui des racines et celle de la largeur des feuilles en fonction de la longueur du pétiole suivant le modèle : $\mathrm{Y}=\alpha+\beta \mathrm{x}_{i}$; Avec $\mathrm{Y}$ étant la valeur observée (variable expliquée) de la plantule $i, \alpha$ le coefficient constant, $\beta$ le coefficient de régression et x les valeurs de la plantule $i$ pour les variables explicatives.

Tableau 1 : Caractéristiques des ethno-variétés selon Sandwidi et al. (2018b).

\begin{tabular}{llll}
\hline Ethno-variété & Fruits & \multicolumn{1}{c}{ Noix } & feuilles et ombrage \\
\hline Taam bonlogo $(\mathrm{Tb})$ & $\begin{array}{l}\text { Petits fruits à pulpe } \\
\text { sucrée }\end{array}$ & $\begin{array}{l}\text { Petite noix de couleur } \\
\text { marron clair }\end{array}$ & $\begin{array}{l}\text { Petites feuilles, à } \\
\text { ombrage peu dense }\end{array}$ \\
& $\begin{array}{l}\text { Fruits longs à pulpe } \\
\text { sucrée }\end{array}$ & $\begin{array}{l}\text { Noix allongée de } \\
\text { couleur marron clair }\end{array}$ & $\begin{array}{l}\text { Feuilles longues à } \\
\text { ombrage dense }\end{array}$ \\
& $\begin{array}{l}\text { Gros fruits à pulpe } \\
\text { abondante et sucrée }\end{array}$ & $\begin{array}{l}\text { Grosse noix de couleur } \\
\text { marron noir }\end{array}$ & $\begin{array}{l}\text { Feuilles courtes à } \\
\text { ombrage moyen }\end{array}$ \\
Taam bèda $(\mathrm{TB})$ & $\begin{array}{l}\text { Fruits ronds à pulpe } \\
\text { moins sucrée }\end{array}$ & $\begin{array}{l}\text { Grosse noix de couleur } \\
\text { rouge vif }\end{array}$ & $\begin{array}{l}\text { Feuilles longues et } \\
\text { effilées à ombrage peu } \\
\text { dense }\end{array}$ \\
& Fruits à pulpe jaune \\
Taam Milissi $(\mathrm{TG})$ & $\begin{array}{l}\text { Grosse noix de couleur } \\
\text { jaunâtre }\end{array}$ & $\begin{array}{l}\text { Feuilles petites à } \\
\text { ombrage peu dense }\end{array}$ \\
\hline
\end{tabular}

Appellations des ethno-variétés en langue locale Mooré. 


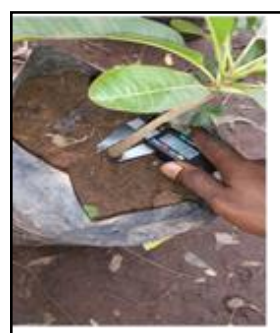

a

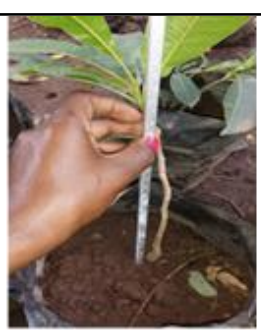

b

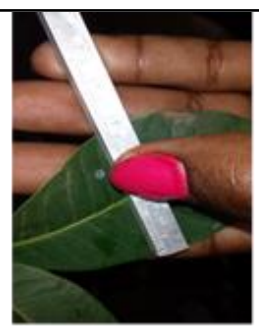

C

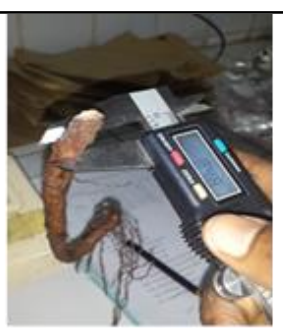

d

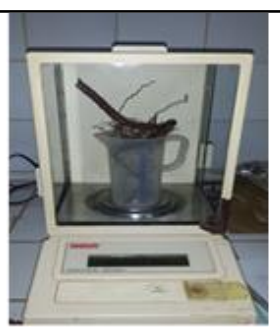

$\mathrm{e}$

a: mesure du diamètre au collet, $\mathbf{b}$ : mesure de la hauteur de la tige, $\mathbf{c}:$ mesure de la largeur de la feuille, $\mathbf{d}$ : mesure du diamètre de la racine principale, e: pesée du poids frais de la racine.

Figure1: Illustrations de quelques mesures biométriques sur les plantules.

\section{RESULTATS}

\section{Caractérisation morphologique des plantules}

\section{Variabilité des caractères morphologiques}

Les analyses de variance sur les paramètres morphologiques ont révélé des différences significatives (Tableau 2). En effet, les différences entre les plantules se situent au niveau de 6 paramètres morphologiques sur les 16 mesurés. Il s'agit notamment du nombre de rameau $(P=0,04)$, du poids sec des feuilles $(P=0,02)$, de la largeur de la feuille $(P=0,03)$, de la longueur de la racine principale $(P=0,0001)$, du poids frais de la racine $(P=0,005)$ et du poids sec des racines $(P=0,014)$.

Le test de Newman Keuls au seuil de 5\% a permis la séparation des moyennes des variables discriminantes au niveau des parties aériennes et souterraines des plantules. Les variables de la partie aérienne (Tableau 3) concernées sont : le poids sec des feuilles et la largeur des feuilles avec des moyennes générales respectives de $3,54 \mathrm{~g}$ et $4,75 \mathrm{~cm}$. En effet, quand on considère la variable poids sec des feuilles, il ressort que les ethno-variétés $\mathrm{Tb}$ et TB portent chacune des feuilles dont le poids à l'état sec est supérieur au poids moyen des feuilles de l'ensemble de l'essai, tandis que les ethno-variétés TM, TT et TG portent chacune des feuilles dont le poids sec est inférieur à cette moyenne. S'agissant de la largeur des feuilles, les ethno-variétés Tb, TB et TM ont des feuilles plus larges que celles de l'ensemble de l'essai, et les ethno-variétés TT et TG ont des feuilles moins larges. Par ailleurs, en considérant les paramètres morphologiques de la partie souterraine, les moyennes générales de l'essai pour les variables longueur de la racine principale, poids frais et poids sec des racines sont respectivement de 52,39 $\mathrm{cm}, 22,94$ et $9,16 \mathrm{~g}$ (Tableau 4). Pour la variable longueur de la racine principale, les ethno-variétés $\mathrm{Tb}, \mathrm{TM}$ et TB se caractérisent par des racines principales longues, et les ethno-variétés TG et TT s'identifient par des racines principales moins longues. En prenant en compte les variables poids frais et sec des racines, les ethnovariétés $\mathrm{Tb}$ et $\mathrm{TM}$ ont chacune des poids de racines (fraiches et sèches) supérieurs au poids moyen de l'essai pour ces variables, et les ethno-variétés TB, TG et TT ont des racines dont le poids est en dessous du poids moyen.

Structuration morphologique des ethnovariétés

L'analyse en composantes principales (ACP) a permis de former des groupes d'ethno-variétés sur la base de la plus proche ressemblance. Les axes principaux F1 et F2 qui expliquent $52,82 \%$ de la variation totale, contiennent plus de la moitié des informations existantes (Figure 2a). La représentation des individus dans le plan formé par ces axes fait ressortir 3 groupes (Figure 2b): le groupe 1 (G1) formé par des ethno-variétés caractérisées par de longues feuilles, 
possédant de longues racines principales et des racines secondaires moins denses. Le deuxième groupe (G2) est formé par les ethno-variétés à longues feuilles et à faible densité de racines secondaires. Le groupe 3 (G3) renferme des ethno-variétés caractérisées par un nombre élevé de feuilles apicales et des racines secondaires denses.

\section{Stratégie d'allocation des ressources}

Pour chaque ethno-variété considérée, la biomasse racinaire est supérieure à celle de la tige et des feuilles (Figure 3). La plus faible biomasse a été observée au niveau des tiges. En effet, les ethno-variétés Tb, TB et TM ont chacune un poids de racines (fraiches et sèches) élevé, suivies des ethno-variétés TG et TT. Mais en considérant les feuilles, les ethno-variétés $\mathrm{TB}$ et $\mathrm{Tb}$ ont les biomasses foliaires fraiches et sèches les plus élevées, suivies des trois autres ethno-variétés. Par contre, elles ont des poids pratiquement identiques au niveau des tiges. Par ailleurs, ces cinq ethno-variétés étudiées ont présenté chacune un rapport Hauteur de la tige/Diamètre au collet inférieure à 7 . Les valeurs maximales et minimales de ce ratio ont été observées respectivement chez les ethno-variétés $\mathrm{Tb}(2,48)$ et TT $(1,25)$. En ce qui concerne le rapport de la biomasse (fraiche ou sèche) aérienne/souterraine, toutes les ethno-variétés ont enregistré chacune une valeur inférieure à 1 (Tableau 5).

\section{Relation entre les caractères morphologiques des plantules}

Les analyses ont permis de mettre en évidence les relations linéaires significatives entre les variables morphologiques des ethnovariétés au stade juvénile. Il s'agit des régressions linéaires significatives entre le poids frais des feuilles et le poids frais des racines mais également entre la largeur de la feuille et la longueur du pétiole (Figure 4).

Tableau 2: Analyse de variance au seuil de 5\% des différentes variables mesurées sur les plantules.

\begin{tabular}{lccc}
\hline Variables & Valeur $\mathbf{F}$ & $\mathbf{P}>\mathbf{F}$ & Moyenne essai \\
\hline Hauteur tige & 2,27 & $0,09 \mathrm{NS}$ & 13,88 \\
Diamètre au collet & 0,41 & $0,79 \mathrm{NS}$ & 7,572 \\
Nombre rameau & 2,89 & $0,04 *$ & 0,6 \\
Poids frais des feuilles & 1,99 & $0,13 \mathrm{NS}$ & 7,64 \\
Poids sec des feuilles & 3,46 & $0,02^{*}$ & 3,54 \\
Nombre de feuille apical & 0,36 & $0,82 \mathrm{NS}$ & 7,56 \\
Largeur de la feuille & 3,23 & $0,03 *$ & 4,75 \\
Longueur de la feuille & 2,08 & $0,12 \mathrm{NS}$ & 12,59 \\
Poids frais de la tige & 1,04 & $0,40 \mathrm{NS}$ & 3,98 \\
Poids sec de la tige & 0,71 & $0,59 \mathrm{NS}$ & 1,65 \\
Longueur de la racine principale & 32,56 & $0,0001^{* * *}$ & 52,39 \\
Diamètre de la racine principale & 1,351 & $0,286 \mathrm{NS}$ & 11,139 \\
Nombre de racines secondaires & 2,143 & $0,112 \mathrm{NS}$ & 3,6 \\
Poids frais des racines & 5,232 & $0,005^{* *}$ & 22,9492 \\
Poids sec des racines & 4,122 & $0,014^{*}$ & 9,163 \\
Longueur du pétiole & 1,089 & $0,389 \mathrm{NS}$ & 2,543 \\
\hline
\end{tabular}

*Différence significative au seuil de 5\%;**Différence très significative au seuil de 1\%;***Différence hautement significative au seuil de $1 \%$, NS : Non Significative. 
Tableau 3: Test de comparaison de Newman Keuls au seuil de 5\% des moyennes des variables de la partie aérienne des plantules.

\begin{tabular}{|c|c|c|c|}
\hline Ethno-variétés & $\begin{array}{c}\text { Nombre de } \\
\text { Rameau }\end{array}$ & $\begin{array}{l}\text { Largeur de la feuille } \\
\text { (cm) }\end{array}$ & $\begin{array}{c}\text { Poids sec des feuilles } \\
\text { (g) }\end{array}$ \\
\hline $\mathrm{Tb}$ & $1,0 \pm 0,07 \mathrm{a}$ & $5,11 \pm 14,57 \mathrm{a}$ & $4,51 \pm 3,1 \mathrm{ab}$ \\
\hline TT & $1,0 \pm 0,0 \mathrm{a}$ & $5,48 \pm 0,96 a b$ & $6,24 \pm 3,69 \mathrm{a}$ \\
\hline TB & $0,2 \pm 0,44 \mathrm{a}$ & $4,59 \pm 0,52 \mathrm{ab}$ & $2,60 \pm 0,85 \mathrm{ab}$ \\
\hline TG & $0,8 \pm 1,09 \mathrm{a}$ & $4,89 \pm 1,07 \mathrm{ab}$ & $3,13 \pm 1,55 \mathrm{ab}$ \\
\hline $\mathrm{TM}$ & $0,0 \pm 0,00 \mathrm{~b}$ & $3,70 \pm 0,98 b$ & $1,20 \pm 0,46 \mathrm{~b}$ \\
\hline Moyenne générale & 0,6 & 4,75 & 3,54 \\
\hline
\end{tabular}

Tableau 4: Test de comparaison de Newman Keuls au seuil de $5 \%$ des moyennes des variables de la partie souterraine des plantules.

\begin{tabular}{|c|c|c|c|}
\hline Ethno-variétés & $\begin{array}{l}\text { Longueur de la racine } \\
\text { principale }(\mathrm{cm})\end{array}$ & $\begin{array}{l}\text { Poids frais des racines } \\
\text { (g) }\end{array}$ & $\begin{array}{l}\text { Poids sec des racines } \\
\text { (g) }\end{array}$ \\
\hline $\mathrm{Tb}$ & $68,4 \pm 4,87 a$ & $37,8 \pm 14,57 \mathrm{a}$ & $14,918 \pm 6 a$ \\
\hline $\mathrm{TT}$ & $37,6 \pm 3,91 \mathrm{c}$ & $18,3 \pm 6,53 b$ & $7,326 \pm 2,31 b$ \\
\hline TB & $54,6 \pm 4,27 b$ & $24,5 \pm 12,51 \mathrm{~b}$ & $10,284 \pm 6,51 \mathrm{ab}$ \\
\hline TG & $41,6 \pm 7,12 c$ & $12,06 \pm 4,15 b$ & $4,7 \pm 1,34 b$ \\
\hline $\mathrm{TM}$ & $59,76 \pm 4,08 \mathrm{~b}$ & $22 \pm 2,65 b$ & $8,588 \pm 1,47 \mathrm{ab}$ \\
\hline Moyenne Générale & 52,39 & 22,94 & 9,16 \\
\hline \multicolumn{4}{|c|}{$\begin{array}{l}\text { Taam tolma }(\mathrm{TT}) ; \text { Taam bonlogo }(\mathrm{Tb}) ; \text { Taam Miidou }(\mathrm{TM}) ; \text { Taam guilissi }(\mathrm{TG}) \text { et Taam bèda }(\mathrm{TB}) ; \pm \text { Ecart-type ; (les } \\
\text { moyennes sur une colonne portant les mêmes lettres ne sont pas significativement différentes au seuil de } 5 \%) \text {. }\end{array}$} \\
\hline Ethno-variété & $\mathbf{H t} / \mathbf{D c}$ & BAf/BSf & BAs/BSs \\
\hline $\mathrm{TB}$ & 1,69 & 0,56 & 0,60 \\
\hline $\mathrm{TM}$ & 1,93 & 0,28 & 0,51 \\
\hline $\mathrm{Tb}$ & 2,48 & 0,50 & 0,54 \\
\hline TG & 1,98 & 0,44 & 0,51 \\
\hline TT & 1,25 & 0,68 & 0,70 \\
\hline
\end{tabular}

Taam tolma (TT); Taam bonlogo (Tb); Taam Miidou (TM); Taam guilissi (TG) et Taam bèda $(\mathrm{TB})$; Hauteur de la tige (Ht) ; Diamètre au collet $(\mathrm{Dc})$; Biomasse aérienne fraiche (BAf); Biomasse souterraine fraiche (BSf) ; Biomasse aérienne sèche (BAs) ; Biomasse souterraine sèche (BSs). 
a) Variables (axes F1 et F2: $52,82 \%$ )

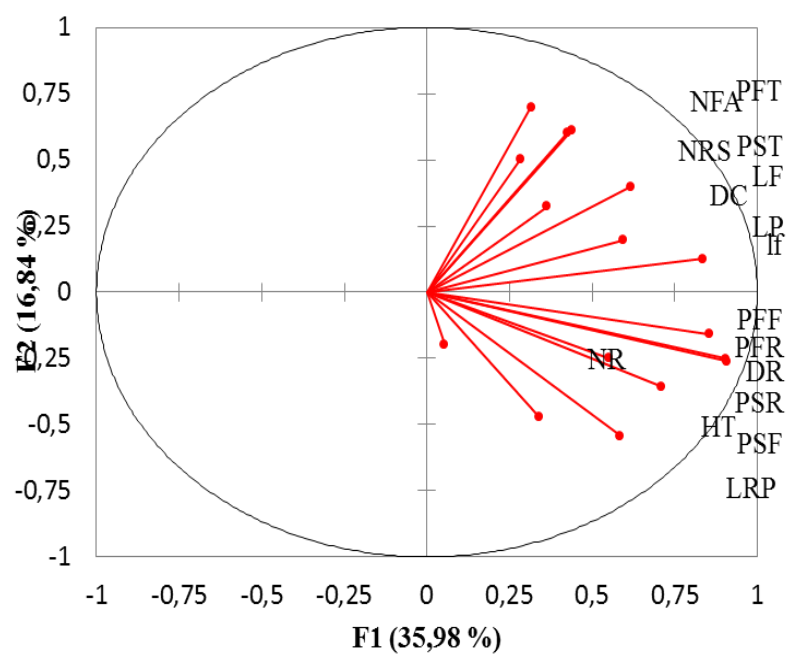

b) Observations (axes F1 et F2 : 52,82 \%)

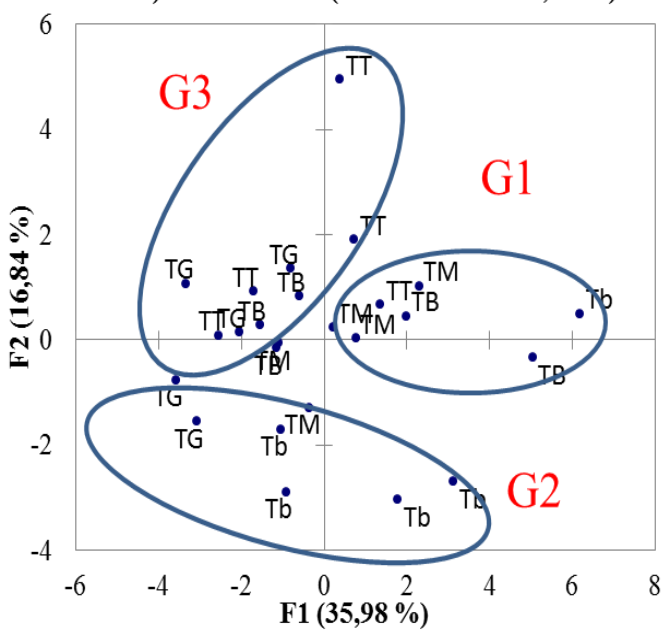

HT: Hauteur de la tige, DC: Diamètre au collet, NR: Nombre de ramifications, PFT: Poids frais de la tige, PST: Poids sec de la tige, LRP: Longueur de la racine principale, DRP: Diamètre de la racine principale, NRS: Nombre de racines secondaires, PFR: Poids frais des racines, PSR: poids sec des racines, LF: Longueur de la feuille, IF: Largeur de la feuille, LP: Longueur du pétiole, PFF: Poids frais des feuilles, PSF: Poids sec des feuilles, TT: Taam tolma, Tb: Taam bonlogo, TM: Taam miidou, TG: Taam guilissi, TB: Taam Bèda.

Figure 2: Structuration morphologique des ethno-variétés selon les axes principaux (F1 et F2).

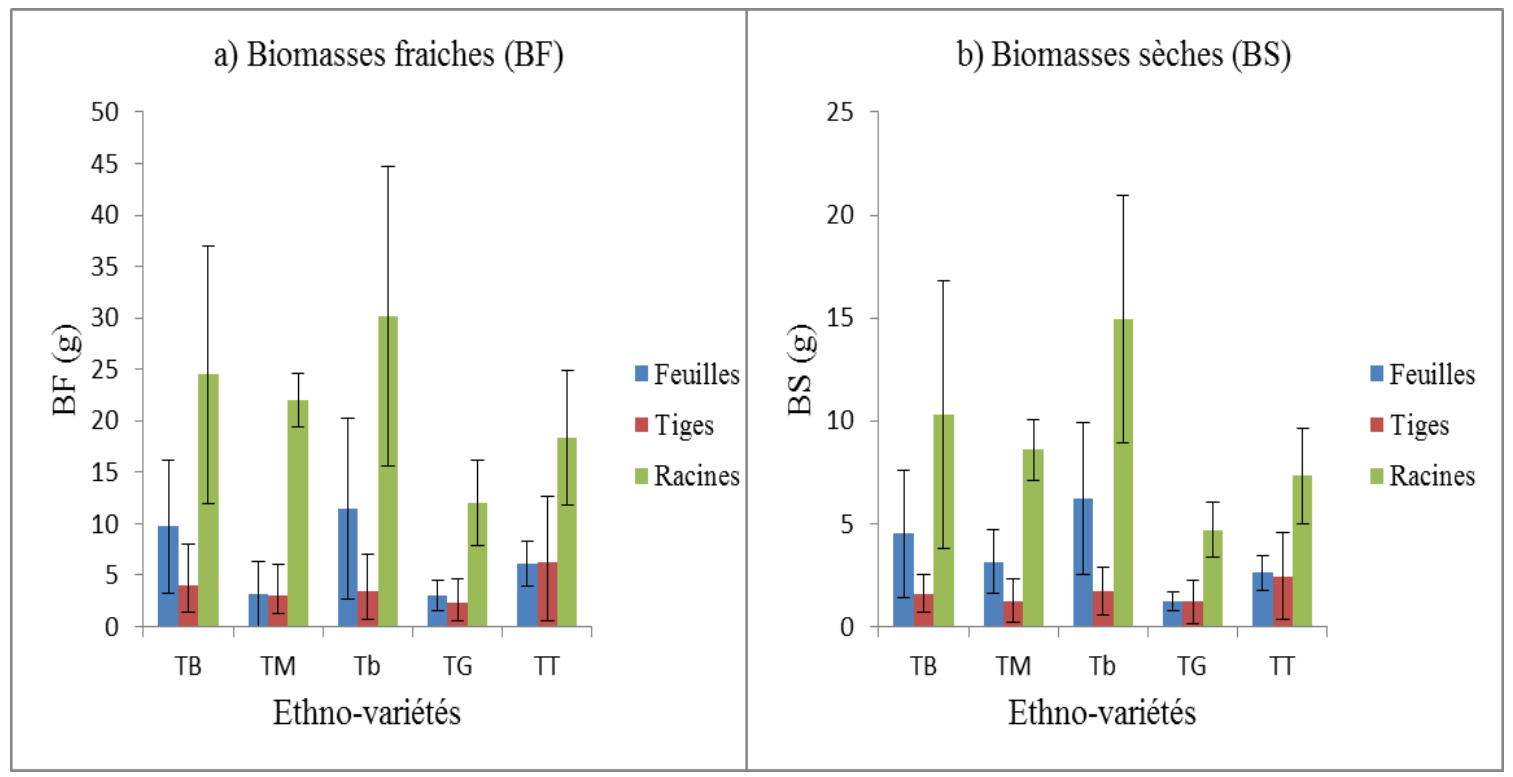

Figure 3: Histogramme de la biomasse fraiche et sèche des cinq ethno-variétés du karité. 

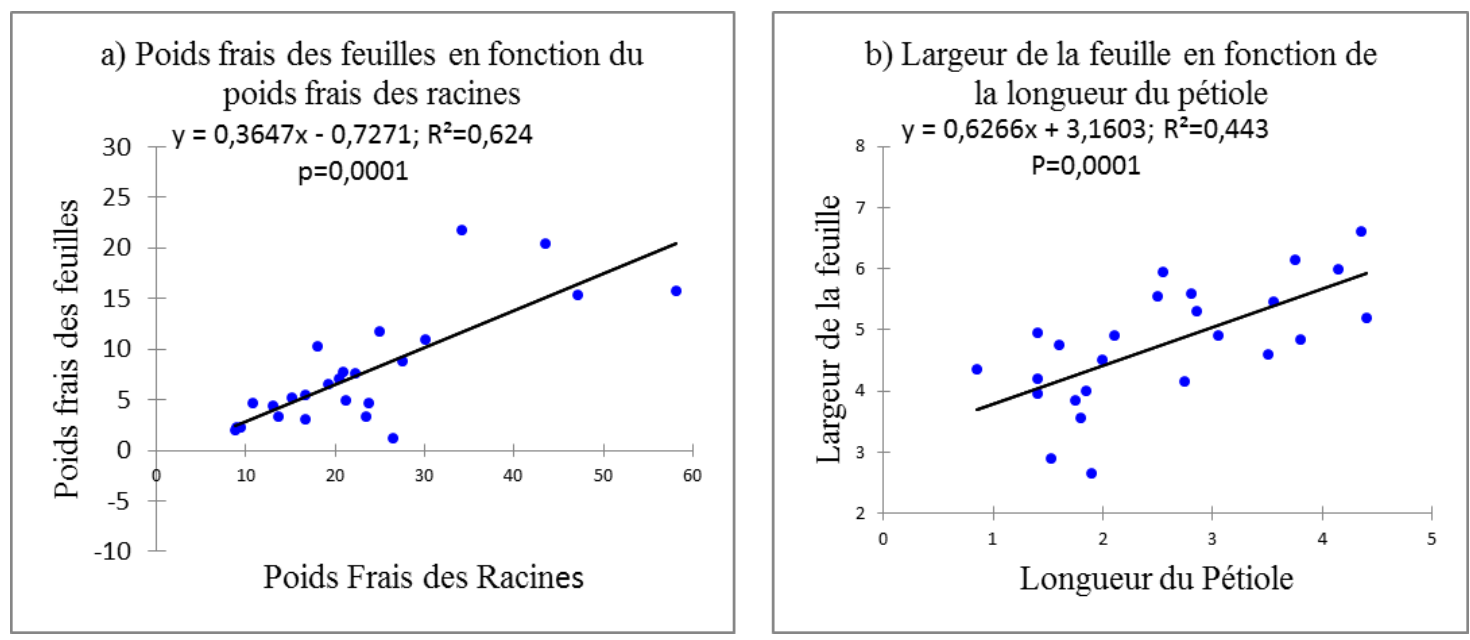

Figure 4: Relations linéaires entre la biomasse foliaire et racinaire; largeur des feuilles et longueur des pétioles.

\section{DISCUSSION}

L'analyse de variance a révélé des différences significatives essentiellement au niveau des caractéristiques des feuilles et des racines. Ces résultats traduisent l'importante variabilité morphologique existante entre les ethno-variétés du karité au stade juvénile. De telles variabilités avaient été observées sur des plantules de karité par Asante et al. (2012) mais également sur des plantules d'autres espèces comme Tamarindus indica (Diallo et al., 2010), Jatropha curcas (Ly et al., 2014) et trois espèces de Chênes du genre Quercus (Sarir et Benmahioul, 2017). D'importantes variabilités avaient été également trouvées au niveau des arbres adultes du karité par Djekota et al. (2014) au Tchad, Souberou et al. (2015) au Benin et Sandwidi et al. (2018a) au Burkina Faso dans un essai de provenances. De plus, des travaux sur les caractères morphologiques (feuilles et capsules) d'autres espèces adultes comme Adansonia digitata L. (Assogbadjo et al., 2006) ont montré des résultats similaires. A l'échelle des feuilles, ces différences sont localisées au niveau de la largeur et du poids. Ces résultats sont en accord avec ceux obtenus par Sandwidi et al. (2018b) sur les ethnovariétés de karité adultes mais également par Kando et al. (2008) sur l'espèce Sclerocarya birrea. En effet, cette différenciation foliaire s'expliquerait par le fait que certaines variétés adaptent les dimensions de leurs feuilles soit pour résister et survivre dans leur environnement, soit pour jouer le rôle d'arrête de la lumière du soleil maximum pour une meilleure photosynthèse (Sarir et Benmahioul, 2017). De plus la largeur des feuilles joue un rôle dans le contrôle de l'évapotranspiration du sol. Ceci explique la difficulté de survie de TM à larges feuilles dans la zone sahélienne et nord soudanien (Sandwidi et al., 2018b). Toutefois si l'on considère les différences observées entre les ethno-variétés à l'échelle de leur partie aérienne, les ethno-variétés Tb, TB et TM possèdent un nombre élevé de rameaux mais également une forte biomasse foliaire. Par contre, les ethno-variétés TT et TG investissent très peu leur croissance pour ces caractères. Ces données signifieraient que les traits morphologiques pourraient servir dans l'identification des ethno-variétés au stade juvénile pour si peu que des corrélations soient établies entre cette morphologie et le comportement de ces ethno-variétés à l'âge adulte (Sandwidi et al., 2018b) . Cela vient confirmer les propos de Kelly et al. (2017) qui ont rapporté que des fermiers Maliens arrivaient à identifier des variantes de karité sur la base des caractéristiques des feuilles. En se rapportant au ratio entre la biomasse (fraiche ou sèche) aérienne et souterraine de 
chaque plantule, il ressort que le karité investit préférentiellement sa croissance au niveau du système racinaire au jeune âge indépendamment de la notion d'ethno-variété. Ce phénomène avait été expliqué chez les espèces ligneuses par Weber et al. (2018) comme étant des stratégies d'adaptations des espèces végétales dans leurs environnements. Il s'agit d'une caractéristique propre au karité qui lui permet de résister aux pressions environnementales telles que la sécheresse, le broutage et les feux de brousse.

En effet, les ethno-variétés Tb, TB et TM investissent plus dans la croissance de leurs pivots (racine principale) que celles des racines secondaires tandis que les ethnovariétés TT et TG investissent essentiellement au niveau de la densification des racines secondaires. Ceci témoigne l'existence d'un compromis dans l'allocation des ressources (les hydrates de carbones) comme l'avait noté Marc-Olivier (2017). La différenciation observée au niveau racinaire pourrait être expliquée comme une stratégie évolutive dont la fonction adaptative est l'exploitation rapide de la nappe phréatique et/ou celles des horizons superficielles (Diallo et al., 2010). De plus, l'ensemble des ethno-variétés ont enregistré chacune un ratio de robustesse (Hauteur/Diamètre) à la norme définie par Lamhamedi et al. (1997). Cela est la preuve que toutes les ethno-variétés étudiées sont caractérisées par une bonne croissance en pépinière. Des résultats similaires avaient été rapportés par Sarir et Benmahioul (2017) sur l'étude de la croissance végétative et du développement des jeunes semis de trois espèces de chênes en pépinière. L'absence de différences significatives entre les ethnovariétés au niveau de la vigueur de croissance (Diamètre au collet et Hauteur totale) montre que ce paramètre n'est pas pertinent dans la différenciation des ethno-variétés au stade juvénile.

Par ailleurs, l'analyse en composantes principales a permis de regrouper les ethnovariétés en trois groupes. Chaque groupe est caractérisé par des descripteurs phénotypiques spécifiques au niveau de la biomasse aérienne et/ou souterraine. Ces résultats corroborent ceux de Diallo et al. (2010) et de Sandwidi et al. (2018b). Cette différenciation pourrait servir d'indice pour anticiper sur la sélection des ethno-variétés désirées en pépinière en fonction des objectifs de productions. Car, chez les espèces ligneuses, au jeune âge les effets maternels sur les descendants sont assez forts pour permettre d'évaluer indirectement les arbres mères (Diallo et al., 2010). Quant aux régressions linéaires, elles ont révélé que la biomasse foliaire est positivement corrélée avec la biomasse racinaire. Aussi, une corrélation positive a été observée entre la largeur des feuilles et la longueur du pétiole. Ces corrélations constitueraient également des indices indispensables dans la discrimination des ethno-variétés au stade juvénile. De telles corrélations avaient été établies par Gwali et al. (2012) et Buba (2013) en Ouganda et au Nigéria respectivement dans les études des sous-espèces paradoxa et nilotica.

\section{Conclusion}

Le présent travail dont l'objectif principal était d'identifier des caractères pertinents pour la sélection précoce des ethnovariétés a mis en évidence des différences entre les ethno-variétés au niveau de la morphologie des feuilles et du système racinaire. Cette grande variabilité observée, peut être exploitée dans les schémas de sélection du karité. En effet, la biomasse foliaire et racinaire, mais également le compromis d'allocation des ressources entre les pivots et la densité des racines secondaires peuvent servir de base dans la discrimination des ethno-variétés. Ils constituent de ce fait des indicateurs pertinents pour la sélection des ethno-variétés du karité au stade juvénile. De cette étude, il ressort que les ethno-variétés $\mathrm{Tb}, \mathrm{TB}$ et TM sont caractérisées par une bonne croissance en longueur du pivot. Elles seraient donc préconisées sur des sols où la nappe phréatique est très profonde tandis que les ethno-variétés TG et TT à racines secondaires denses auront de préférence les parties superficielles du sol et seront adaptées à des sols peu filtrants. Ces informations collectées sur les plantules constituent des données de base pour évaluer en amont les 
arbres mères et en aval les arbres en plantation lorsque les plantules seront soumises aux effets de l'environnement.

En dépit de l'intérêt de cette étude, le suivi de ces plantules en plantation sur plusieurs années serait nécessaire afin de permettre de déterminer l'âge propice de la sélection en milieu réel à travers des tests d'héritabilité qui seront calculés périodiquement. Il serait également important d'établir des relations entre les caractéristiques du système racinaire et les gènes responsables du phénotype observé par la méthode des QTL (Quantitative Trait Locis).

\section{CONFLIT D'INTERETS}

Les auteurs de ce manuscrit déclarent qu'il n'y a pas de conflit d'intérêts pour cet article.

\section{CONTRIBUTIONS DES AUTEURS}

Le sujet a été proposé par BOD, la rédaction et l'exécution du protocole ont été assurées par AS et MSBD, les analyses statistiques des données et la rédaction du manuscrit ont été réalisées par AS sous la supervision de BOD et MS.

\section{REMERCIEMENTS}

Cet article a été rendu possible grâce au soutien financier accordé par l'Ambassade du Royaume de Danemark au Burkina Faso dans la mise en œuvre du projet " Restauration des peuplements et l'amélioration de la production du karité au Burkina Faso».

\section{REFERENCES}

Asante WJ, Banidiyia MA, Tom-Dery D. 2012. Effect of planting depth on the germination and initial growth and development of shea (Vitellaria paradoxa C.F. Gaertn). International Journal of Biosciences, 2(12):146-152.

Assogbadjo AE, Kyndt T, Sinsin V, Gheyseng G, Van damme P. 2006. Patterns of genetic and morphometric diversity in Baobab (Adansonia digitata) population across different climatic zones of Benin
(West Africa). Annal of Botany, 97(5): 819-830.

DOI: https://doi.org/10.1093/aob/mcl043

Badini Z, Kaboré M, Van der Mheen-Sluijer J, Vellema S. 2011. Le marché du karité et ses évolutions. VC4PD. Research Paper, 12: 34p.

Bayala J, Ouédraogo SJ, Ong CK. 2009. Early growth performance and water use of planted West African provenances of Vitellaria paradoxa Gaertn. C.F. (karité) in Gonsé, Burkina Faso. Agroforestry Systems, $\quad \mathbf{7 5}(2)$ : 117-127. DOI https://doi.org/10.1007/s10457-0089167-9.

Belem B, Olsen CS, Theilade RB, Guinko S, Lykke AM, Diallo A, Boussim IJ. 2008. Identification des arbres hors forêt préférés des populations du Sanmatenga (Burkina Faso). Revue Bois et Forêts des tropiques, 298(4): 53-64.

Buba T. 2013. Relationships between stem diameter at breast height $(\mathrm{DBH})$, tree height, crown length, and crown ratio of Vitellaria paradoxa C.F. Gaertn in the Nigerian Guinea Savanna. African Journal of Biotechnology, 12(22): 34413446. DOI: $10.5897 / A J B 12.463$

Bezzalla A, Boudjabi S, Chenchouni H. 2018. Seedlings of Argan (Argania spinosa) from different geographical provenances reveal variable morphological growth responses to progressive drought stress under nursery conditions. Agroforestry Systems, 92(5): 1201-1211. DOI: https://doi.org/10.1007/s10457-0160057-2.

Devineau JL, Fournier A, Nignan S. 2009. "Ordinary biodiversity" in western Burkina Faso (West Africa): what vegetation do the state forests conserve? Biodivers. Conserv., 18(8): 2075-2099. DOI : https://doi.org/10.1007/s10531008-9574-2.

Diallo BO. 2001. Biologie de la reproduction et évaluation de la diversité génétique chez une légumineuse: Tamarindus indica $\mathrm{L}$. (Caesalpinoïdae). Thèse de Doctorat, Science et Technique du Languedoc, Université Montpellier II, France, 119p. 
Diallo BO, Joly IH, Hossaert-McKey M, McKey D, Chevallier MH. 2007. Genetic diversity of Tamarindus indica populations: Any clues on the origin from its current distribution? African Journal of Biotechnology, 6(7): 853-860.

Diallo BO, Joly IH, Hossaert-McKey M, McKey D, Chevallier MH. 2010. Variation des caractères biométriques de neuf provenances de Tamarindus indica L. (Caesalpinioideae). Fruits, 65(2): 153167. DOI: https://doi.org/10.1051/fruits/2010010

Djekota C, Diouf D, Sane S, Mbaye MS, Noba K. 2014. Morphological characterization of shea tree (Vitellaria paradoxa subsp. paradoxa) populations in the region of Mandoul in Chad. Int. J. Biodiv. Conserv., 6(2): 184-193. DOI: https://doi.org/10.5897/ IJBC2013.0662

Gwali S, Okullo JBL, Eilu G, Nakabonge G, Nyeko P, Vuzi P. 2011. Folk classification of Shea butter tree (Vitellaria paradoxa subsp. nilotica) ethno-varieties in Uganda. Ethnobot. Res. Appl., 9: 243-256. DOI: https://doi.org/10.17348/era.9.0.243-256

Gwali S, Nakabonge G, Okullo JBL, Eilu G, Nyeko P, Vuzi P. 2012. Morphological variation among Shea tree (Vitellaria paradoxa Subsp. nilotica) ethno-varieties in Uganda. Genetic Resources and Crop Evolution, 59(8): 1883-1898. DOI: https://doi.org/10.1007/s10722-0129905-8.

Karambiri M, Elias M, Vinceti B, Grosse A. 2017. Exploring local knowledge and preferences for Shea (Vitellaria paradoxa) ethnovarieties in Southwest Burkina Faso through a gender and ethnic lens. For. Trees and Livelihoods, 26(1):13-28.

DOI: https://doi.org/10.1080/14728028.2016.1 236708

Kando P, Zongo JD, Nanema RK, Traore ER. 2008. Etude de la variation de quelques carac- tères morphologiques d'un échantillon de Sclerocarya birrea au Burkina Faso. Int. J. Biol. Chem. Sci., 2(4): 549-562.
Kelly BA, Senou O. 2017. Variation of leaf and fruit characteristics of Vitellaria paradoxa (shea tree) according to agronomical performance along southnorth climatic gradient in Mali. African Journal of Plant Science, 11(5):142-150. DOI: 10.5897/AJPS2017.1536

Kobe RK, Lyer M, Walters MB. 2010. Optimal partitioning theory revisited: nonstructural carbohydrates dominate root mass responses to nitrogen. Ecology, $\quad$ 91(1): $\quad 166-179$. DOI:10.1890/09-0027.1

Korbo A, Sanou H, Ræbild A, Jensen JS, Hansen JK, Kjær $\quad$ ED. 2012. Comparison of East and West African populations of baobab (Adansonia digitata L.). Agroforestry Systems, 85(3): 505-518. DOI: https://doi.org/10.1007/s10457-0119464-6

Lamhamedi M, Fortinn S, Ortinn JA, Ammari Y, Ben Jalloun S, Poirier M, Fecteau B, Bougacha A, Godin L. 1997. Évaluation des composts, des substrats et de qualité des plants (Pinus pinea, Punis halepensis, Cupressus sumpervirens et Quercus suber) élevés en conteneurs. Projet Bird 3601, Rapport technique : Exécution des travaux d'aménagement de trois pépinières pilotes en Tunisie, Direction Générale des Forêts, Tunisie et Pampev Internationale Ltée, Canada, $121 \mathrm{p}$.

Lamien N, Kabore AK, Tamini Z. 2008. Greffage de quatre fruitiers locaux : (Tamarindus indica L., Saba senegalensis (A. DC.) Pichon, Lannea microcarpa Engl. et K. Krause et Sclerocarya birrea (A. Rich.) Hochst au Burkina Faso. Science et technique, Sciences naturelles et agronomie, $\mathbf{3 0}(1)$ : 53-60.

Ly MO, Kumar D, Diouf M, Nautiyal S, Diop T. 2014. Effet de la salinité sur la croissance et la production de biomasse de deux provenances de Jatropha curcas L. cultivés en serre Mamadou. Int. J. Biol. Chem. Sci., 8(1): 46-56. DOI: http://dx.doi.org/10.4314/ijbcs.v8i1.5 
Marc-olivier M. 2017. Effets de la diversité sur la croissance de jeunes arbres de la forêt tempérée: rôle de la lumière et stratégies de distribution de la biomasse. Mémoire de maîtrise en biologie, Université du Québec, Montréal, 92p.

Poorter H, Niklas KJ, Reich PB, Oleksyn J, Poot P, Mommer L. 2012. Biomass allocation to leaves stems and roots: meta-analysis of interspecific variation and environmental control. New Phytologist, 193: 30-50. DOI:10.1111/j.1469-8137.2011.03952.x.

Ræbild A, Larsen AS, Jensen S, Ouedraogo M, Groote SD, Damme PV, Bayala J, Diallo BO, Sanou H, Kalinganire A, Kjaer ED. 2011. Advances in domestication of indigenous fruit trees in the West African Sahel. New Forests, 41: 297-315. DOI : 10.1007/s11056-0109237-5

Sandwidi A, Diallo BO, Sawadogo M. 2018. Caractérisation morphologique de cinq écotypes du karité dans un essai de provenances au Burkina Faso. Science et Technique, Sciences Naturelles et Appliquées, 4: 465-478.

Sandwidi A, Diallo BO, Lamien N, Vinceti B, Sanon K, Coulibaly P, Paré S, Sawadogo M. 2018. Participatory identification and characterisation of shea butter tree (Vitellaria paradoxa C.F. Gaertn.) ethnovarieties in Burkina Faso. Fruits, 73(3) : $\quad 141-152 . \quad$ DOI : https://doi.org/10.17660/th2018/73.3.1

Sarir R, Benmahioul B. 2017. Etude comparative de la croissance végétative et du développement de jeunes semis de trois espèces de chênes (chêne vert, chêne liège et chêne zéen) cultivés en pépinière. Agriculture and Forestry Journal, 1(2): 42-48. $\quad$ DOI: http://ojs.univtlemcen.dz/index.php/AFJ/.

Souberou TK, Ahoton EL, Ezin V, Hamidou Seko E. 2015. Agro-morphological variability of shea populations (Vitellaria paradoxa C.F Gaertn) in the Township of Bassila, Benin Republic. Journal of Plant Breeding and Crop Science, 7(2):28-37. DOI: 10.5897/JPBCS2014.0451

Thiombiano DNE, Dibong SD, Lamien N, Boussim IJ. 2010. Etat des peuplements des espèces ligneuses de soudure des communes rurales de Pobé-Mangao et de Nobéré (Burkina Faso). Journal of Animal and Plant Sciences, 9(1): 11041116.

Traore L. 2008. Inventaire des espèces ligneuses utilitaires de la région SudOuest du Burkina Faso et état des populations de trois espèces à haute valeur économique. Mémoire de DEA en Sciences Biologiques Appliquées. Université de Ouagadougou, Burkina Faso, 46p.

Weber JC, Montes CS, Soumana I, Diallo BO, Abasse T, Larwanou M, Bationo AB. 2018. Genetic and geographic variation in growth of Balanites aegyptiaca in Niger: comparing results from provenance/ progeny tests in the nursery and field. New Forests. DOI : https://doi.org/10.1007/s11056-0189686-9 